\title{
A One-Field Discontinuous Galerkin Formulation of Non-Linear Kirchhoff-Love Shells
}

\author{
Ludovic Noels \\ Aerospace and Mechanical Engineering Department - Computational \& Multiscale Mechanics of Materials \\ (CM3), University of Liège, Chemin des Chevreuils 1, 4000 Liège, \\ Belgium
}

12th International ESAFORM Conference on Material Forming

27 - 29 April 2009 University of Twente

The Netherlands, 4 pages 


\title{
A ONE-FIELD DISCONTINUOUS GALERKIN FORMULATION OF NON-LINEAR KIRCHHOFF-LOVE SHELLS
}

\author{
L. Noels* \\ University of Liège - Computational \& Multiscale Mechanics of Materials
}

\begin{abstract}
Spatially-discontinuous Galerkin methods constitute a generalization of weak formulations, which allow for discontinuities of the problem unknowns in its domain interior. This is particularly appealing for problems involving high-order derivatives, since discontinuous Galerkin (DG) methods can also be seen as a means of enforcing higher-order continuity requirements. Recently, DG formulations of linear and non-linear Kirchhoff-Love shell theories have been proposed. This new one-field formulations take advantage of the weak enforcement in such a way that the displacements are the only discrete unknowns, while the $\mathrm{C} 1$ continuity is enforced weakly. The Resulting one field formulation is a simple and efficient method to model thin structures and can be applied to various computational methods.
\end{abstract}

KEYWORDS: Kirchhoff-Love shells, non-linear mechanics, discontinuous Galerkin methods

\section{INTRODUCTION}

When modeling thin structures, a finite-element formulation without rotation degree of freedom simplifies the implementation, which can be appealing when dealing with computational homogenization of thin structures [1]. Kirchhoff-Love assumption for shells constrains the surface normal to remain perpendicular to the shell. This omission of the shearing allows the formulation of the problem as a one-field displacement method, but leads to weak formulations involving high order derivatives. These high-order derivative terms require polynomial approximations of the displacement field with the same degree of continuity, which, for finite-elements methods, corresponds to the use of shape-functions fulfilling the high-order continuity requirements.

A solution is to enforce weakly the high-order continuity requirements by recourse to DG methods. This weak enforcement corresponds to allow for jumps at the interelement boundaries, while consistency and stability of the formulation are ensured by boundary integral terms. If, in the context of solid mechanics, DG can be developed for problems involving discontinuities in the unknown field, see [2-5] for non-linear solid mechanics, but it has also been exploited in the case of $\mathcal{C}^{0}$ displacement unknown fields, which suffer from discontinuities in their derivative. This method has been exploited for applications to beams and plates [6-8], and more recently for linear and non-linear Kirchhoff-Love shells [9, 10]. In this resulting one-field formulation, the jump discontinuities are related to the derivatives of the continuous unknown field.

Consistency, optimal convergence rate and stability of this DG weak form are ensured by the addition of the integration on the element-boundaries of respectively the resul-

\footnotetext{
*Corresponding author: Chemin des Chevreuils 1, B-4000 Liège, Belgium, L.Noels@ulg.ac.be
}

tant moment, its symmetric counterpart and a (sufficiently large) quadratic term. This method was also shown to reduce locking inherent to finite-element discretizations, especially for thin structures like beams, plates or shells, for which the locking results in excessive stiffness when the membrane and bending modes are mixed.

\section{KIRCHHOFF-LOVE SHELLS}

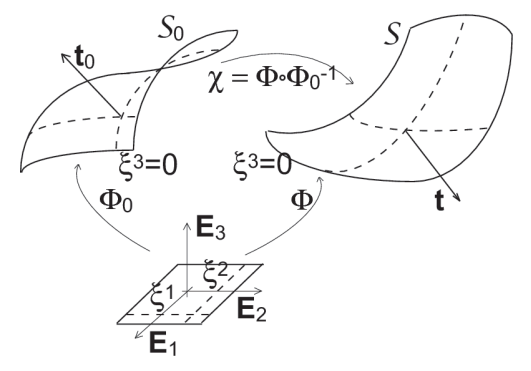

Figure 1: Description of the different configurations of the shell.

A thin body can be described by considering its midsurface section as a Cosserat plane $\left(\xi^{1}, \xi^{2}\right) \in \mathcal{A}_{0}$ and a third coordinate, representing the thickness, and belonging to the interval $\xi^{3} \in\left[h_{\min 0} ; h_{\max 0}\right]$. The representation of the body in the inertial frame is illustrated in Fig. 1. A configuration $\mathcal{S}$ of the shell is described by using $\varphi\left(\xi^{1}, \xi^{2}\right)$ the mapping of the mid-surface and by the unit vector $t$, which is the director of the midsurface $(\|\boldsymbol{t}\|=1)$. Therefore, the position $\boldsymbol{x}$ of the points in this configuration $\mathcal{S}$ can be defined by the mapping $\boldsymbol{x}=\boldsymbol{\Phi}\left(\xi^{I}\right)=\boldsymbol{\varphi}\left(\xi^{\alpha}\right)+\xi^{3} \lambda_{h} \boldsymbol{t}\left(\xi^{\alpha}\right)$, with $\lambda_{h}$ the thickness stretch of the shell resulting from the Cosserat plane deformation. The transformation $\chi=\Phi \circ \boldsymbol{\Phi}_{0}^{-1}$ between 
two configurations is characterized by the two-point deformation gradient

$$
\mathbf{F}=\boldsymbol{\nabla} \boldsymbol{\Phi} \circ\left[\boldsymbol{\nabla} \boldsymbol{\Phi}_{0}\right]^{-1}
$$

with $\boldsymbol{\nabla} \boldsymbol{\Phi}=\boldsymbol{g}_{i} \otimes \boldsymbol{E}^{i}$ when defined in the reference frame. The Jacobian related to the deformation of the mid-surface is computed by

$$
\bar{J}=\frac{\bar{j}}{\bar{j}_{0}} \text {, with } \bar{j}=\left\|\boldsymbol{\varphi}_{, 1} \wedge \boldsymbol{\varphi}_{, 2}\right\| .
$$

Since the particular case of Kirchhoff-Love shells corresponds to neglect shearing deformations, the unit vector $\boldsymbol{t}$ remains always perpendicular to $\varphi, \alpha$ with

$$
t=\frac{\varphi_{, 1} \wedge \varphi_{, 2}}{\left\|\varphi_{, 1} \wedge \varphi_{, 2}\right\|}
$$

Following [11], the governing equations of a thin body are obtained by integrating on the thickness the equations of force and moment equilibrium, leading to

$$
\begin{aligned}
\frac{1}{\bar{j}}\left(\bar{j} \boldsymbol{n}^{\alpha}\right)_{, \alpha}+\boldsymbol{n}^{\mathcal{A}_{0}}=0 \quad \text { on } \mathcal{A}_{0} \text { and } \\
\frac{1}{\bar{j}}\left(\bar{j} \tilde{\boldsymbol{m}}^{\alpha}\right)_{, \alpha}-\boldsymbol{l}+\lambda \boldsymbol{t}+\tilde{\boldsymbol{m}}^{\mathcal{A}_{0}}=0 \quad \text { on } \mathcal{A}_{0},
\end{aligned}
$$

where, $\boldsymbol{n}^{\alpha}$ is the resultant stress vector, $\tilde{\boldsymbol{m}}^{\alpha}$ is the resultant torque vector, $l$ is the resultant across-the-thickness stress vector, $\lambda$ is an undefined pressure, where $n^{\mathcal{A}_{0}}$ is the resultant external surface traction and where $\tilde{\boldsymbol{m}}^{\mathcal{A}_{0}}$ is the resultant external torque by unit surface.

The resultant vectors can be obtained from any computational method. For example, assuming an homogeneous structure under the plane stress assumption, they can be evaluated from a Simpson integration in terms of the Kirchhoff stress tensor $\tau$, leading to

$$
\begin{aligned}
\boldsymbol{n}^{\alpha} & =\frac{1}{\bar{j}} \int_{h_{\min 0}}^{h_{\max 0}} \tau^{\beta \alpha} \boldsymbol{g}_{\beta} \operatorname{det}\left(\nabla \boldsymbol{\Phi}_{0}\right) d \xi^{3} \\
\tilde{\boldsymbol{m}}^{\alpha} & =\frac{1}{\bar{j}} \int_{h_{\min 0}}^{h_{\max 0}} \xi^{3} \tau^{\beta \alpha} \boldsymbol{g}_{\beta} \operatorname{det}\left(\boldsymbol{\nabla} \boldsymbol{\Phi}_{0}\right) d \xi^{3}, \text { and }(7) \\
\boldsymbol{l} & =\frac{1}{\bar{j}} \int_{h_{\min 0}}^{h_{\max 0}} \tau^{i 3} \boldsymbol{g}_{i} \operatorname{det}\left(\boldsymbol{\nabla} \boldsymbol{\Phi}_{0}\right) d \xi^{3}=0
\end{aligned}
$$

This set of governing equations is accompanied by boundary conditions applied on the boundary $\partial \mathcal{A}_{0}$ of the midsurface $\mathcal{A}_{0}$. This boundary $\partial \mathcal{A}_{0}$ is decomposed into a part $\partial_{T} \mathcal{A}_{0}$ where the unit vector is constraint to $t=\bar{t}$ and into a part $\partial_{M} \mathcal{A}_{0}$ where the applied torque is constraint to $\tilde{\boldsymbol{m}}^{\alpha} \nu_{\alpha}=\overline{\tilde{\boldsymbol{m}}}$. Similarly, the boundary $\partial \mathcal{A}_{0}$ is also decomposed into a part $\partial_{U} \mathcal{A}_{0}$ where the position is constraint to $\varphi=\bar{\varphi}$ and into a part $\partial_{N} \mathcal{A}_{0}$ where the traction is constrained to $\boldsymbol{n}^{\alpha} \nu_{\alpha}=\overline{\boldsymbol{n}}$. In these equations, $\nu_{\alpha}$ are the component of the external normal in the dual convected basis $\varphi^{, \alpha}$.

\section{DISCONTINUOUS GALERKIN FOR- MULATION}

In this section, a framework for numerical approximation of the shell equations described above based on a $\mathcal{C}^{0}$ polynomial approximation of the unknown field $\varphi$ is proposed. In this formulation, the resulting discontinuity in the surface director $t$ is accounted for using a new discontinuous Galerkin formulation.

\subsection{WEAK FORMULATION OF THE PROBLEM}

At this point, the mid-surface $\mathcal{A}_{0}$ is approximated by a discretization $\mathcal{A}_{h}$ into finite-elements $\mathcal{A}_{e}$ of $\mathcal{A}_{0} \simeq \mathcal{A}_{h}=$ $\bigcup_{e} \overline{\mathcal{A}}_{e}$. The boundary $\partial \mathcal{A}_{e}$ of an element $\mathcal{A}_{e}$ can be common with the boundary of $\mathcal{A}_{h}$, leading to the boundary parts $\partial_{U} \mathcal{A}_{e}, \partial_{T} \mathcal{A}_{e}, \partial_{M} \mathcal{A}_{h}$, and $\partial_{N} \mathcal{A}_{e}$. The remaining part of the boundary $\partial \mathcal{A}_{e}$ is shared with another finite element and is part of the interior boundary $\partial_{I} \mathcal{A}_{h}$, with

$$
\partial_{I} \mathcal{A}_{e}=\partial \mathcal{A}_{e} \backslash \partial \mathcal{A}_{h}, \text { and } \partial_{I} \mathcal{A}_{h}=\bigcup_{e} \partial \mathcal{A}_{e} \backslash \partial \mathcal{A}_{h}
$$

Instead of seeking the exact solution $\varphi$, a polynomial approximation $\varphi_{h}$ constitutes the solution to the finite element problem. In this work, a continuous polynomial approximation is considered, but the derivatives of the displacement field are allowed to be discontinuous on the element boundaries. Therefore, both the displacement field $\varphi_{h}$ and the test functions $\delta \varphi$ are continuous across element-interfaces but allow for jump discontinuities in their derivative, which has to be accounted for when establishing the new weak form of the problem.

Multiplying Eq. (4) by a test function $\delta \varphi$ and Eq. (5) by the corresponding variation of unit vector $\lambda_{h} \delta \boldsymbol{t}=$ $\lambda_{h} \boldsymbol{t}(\delta \boldsymbol{\varphi})$, state the problem as finding $\varphi_{h}$ such that

$$
\begin{gathered}
0=\sum_{e} \int_{\overline{\mathcal{A}}_{e}}\left(\bar{j} \boldsymbol{n}^{\alpha}\left(\boldsymbol{\varphi}_{h}\right)\right)_{, \alpha} \cdot \delta \boldsymbol{\varphi} d \mathcal{A}_{0}+ \\
\sum_{e} \int_{\overline{\mathcal{A}}_{e}}\left[\left(\bar{j} \tilde{\boldsymbol{m}}^{\alpha}\left(\boldsymbol{\varphi}_{h}\right)\right)_{, \alpha}-\bar{j} \boldsymbol{l}\right] \cdot \delta \boldsymbol{t} \lambda_{h} d \mathcal{A}_{0}+ \\
\int_{\mathcal{A}_{h}} \boldsymbol{n}^{\mathcal{A}_{0}} \cdot \delta \boldsymbol{\varphi} \bar{j} d \mathcal{A}_{0}+\int_{\mathcal{A}_{h}} \tilde{\boldsymbol{m}}^{\mathcal{A}_{0}} \cdot \delta \boldsymbol{t} \lambda_{h} \bar{j} d \mathcal{A}_{0} .
\end{gathered}
$$

The integration by parts of these integrals followed by the application of the Gauss theorem leads to

$$
\begin{gathered}
0=-\sum_{e} \int_{\overline{\mathcal{A}}_{e}} \bar{j} \boldsymbol{n}^{\alpha}\left(\boldsymbol{\varphi}_{h}\right) \cdot \delta \boldsymbol{\varphi}_{, \alpha} d \mathcal{A}_{0}+ \\
\sum_{e} \int_{\partial \mathcal{A}_{e}} \bar{j} \boldsymbol{n}^{\alpha}\left(\boldsymbol{\varphi}_{h}\right) \cdot \delta \boldsymbol{\varphi} \nu_{\alpha} d \mathcal{A}_{0}- \\
\sum_{e} \int_{\overline{\mathcal{A}}_{e}} \bar{j} \tilde{\boldsymbol{m}}^{\alpha}\left(\boldsymbol{\varphi}_{h}\right) \cdot\left(\delta \boldsymbol{t} \lambda_{h}\right)_{, \alpha} d \mathcal{A}_{0}+ \\
\sum_{e} \int_{\partial \mathcal{A}_{e}} \bar{j} \tilde{\boldsymbol{m}}^{\alpha}\left(\boldsymbol{\varphi}_{h}\right) \cdot \delta \boldsymbol{t} \lambda_{h} \nu_{\alpha} d \mathcal{A}_{0}- \\
\sum_{e} \int_{\overline{\mathcal{A}}_{e}} \bar{j} \boldsymbol{l} \cdot \delta \boldsymbol{t} \lambda_{h} d \mathcal{A}_{0}+ \\
\int_{\mathcal{A}_{h}} \tilde{\boldsymbol{m}}^{\mathcal{A}_{0}} \cdot \delta \boldsymbol{t} \lambda_{h} \bar{j} d \mathcal{A}_{0}+\int_{\mathcal{A}_{h}} \boldsymbol{n}^{\mathcal{A}_{0}} \cdot \delta \boldsymbol{\varphi} \bar{j} d \mathcal{A}_{0} .
\end{gathered}
$$

Variation $\delta \lambda_{h}$ is omitted as it corresponds to the plane stress or plane strain assumption.

When analyzing the boundary integrals in Eq. (11), it appears that the contribution involving the scalar product 
with $\delta \varphi$ has the same meaning as for continuous Galerkin methods. Indeed, since $\delta \varphi \in \mathcal{C}^{0}\left(\mathcal{A}_{h}\right)$, and since for the exact solution $\boldsymbol{n}^{\alpha}$ is also continuous, the following substitution satisfies the consistency requirement:

$$
\begin{array}{r}
\sum_{e} \int_{\partial \mathcal{A}_{e}} \bar{j} \boldsymbol{n}^{\alpha}\left(\boldsymbol{\varphi}_{h}\right) \cdot \delta \boldsymbol{\varphi} \nu_{\alpha} d \mathcal{A}_{0} \rightarrow \\
\int_{\partial \mathcal{A}_{h}} \bar{j} \boldsymbol{n}^{\alpha}\left(\boldsymbol{\varphi}_{h}\right) \cdot \delta \boldsymbol{\varphi} \nu_{\alpha} d \mathcal{A}_{0} .
\end{array}
$$

By contrast, the contribution involving the tensorial product with $\delta \boldsymbol{t}$ requires particular attention since it is discontinuous across interelement boundaries. Since only the $\mathcal{C}^{0}$ continuity is ensured across $\partial_{I} \mathcal{A}_{h}$, jump $\llbracket \bullet \rrbracket$ and mean $\langle\bullet\rangle$ operators are defined on this boundary, with

$$
\begin{aligned}
\llbracket \bullet \rrbracket & =\bullet^{+}-\bullet^{-}, \text {and } \\
\langle\bullet\rangle & =\frac{1}{2}\left(\bullet^{+}+\bullet^{-}\right) .
\end{aligned}
$$

It is worth noticing that if definition (13) of the jump operator is not independent of the choice of the + and - sides of an element edge, when this jump is used in combination with the outward unit normal of the - element $\nu^{-}$, the formulation becomes consistent and independent on this choice. Although jump and mean operators are meaningful on the interior boundary $\partial_{I} \mathcal{A}_{0}$, jump definition can be extended on $\partial_{T} \mathcal{A}_{0}$ as a way of enforcing weakly the boundary conditions, see [10]. From these definitions, the boundary term dependent on $\delta \boldsymbol{t}$ is rewritten

$$
\begin{aligned}
& \sum_{e} \int_{\partial_{I} \mathcal{A}_{e}} \bar{j} \tilde{\boldsymbol{m}}^{\alpha}\left(\boldsymbol{\varphi}_{h}\right) \cdot \delta \boldsymbol{t} \lambda_{h} \nu_{\alpha} d \mathcal{A}_{0}= \\
& -\int_{\partial_{I} \mathcal{A}_{h}} \llbracket \bar{j} \tilde{\boldsymbol{m}}^{\alpha}\left(\boldsymbol{\varphi}_{h}\right) \cdot \delta \boldsymbol{t} \lambda_{h} \rrbracket \nu_{\alpha}{ }^{-} d \mathcal{A}_{0} .
\end{aligned}
$$

The main idea of the discontinuous Galerkin method is to address the contribution of the inter-element discontinuity terms by introducing a numerical flux $\boldsymbol{h}\left(\left(\bar{j} \lambda_{h} \tilde{\boldsymbol{m}}^{\alpha}\right)^{+},\left(\bar{j} \lambda_{h} \tilde{\boldsymbol{m}}^{\alpha}\right)^{-}, \nu_{\alpha}^{-}\right)$, chosen equal to $\nu_{\alpha}^{-}\left\langle\bar{j} \lambda_{h} \tilde{\boldsymbol{m}}^{\alpha}\right\rangle$ in the present work, which leads to the substitution

$$
\begin{array}{r}
\int_{\partial_{I} \mathcal{A}_{h}} \llbracket \bar{j} \tilde{\boldsymbol{m}}^{\alpha}\left(\boldsymbol{\varphi}_{h}\right) \cdot \delta \boldsymbol{t} \lambda_{h} \rrbracket \nu_{\alpha}^{-} d \mathcal{A}_{0} \rightarrow \\
\int_{\partial_{I} \mathcal{A}_{h}} \llbracket \delta \boldsymbol{t} \rrbracket \cdot\left\langle\bar{j} \lambda_{h} \tilde{\boldsymbol{m}}^{\alpha}\right\rangle \nu_{\alpha}^{-} d \mathcal{A}_{0} .
\end{array}
$$

Combining Eqs. (12) to (16) allows rewriting the weak form (11) as

$$
\begin{aligned}
& \int_{\mathcal{A}_{h}} \bar{j} \boldsymbol{n}^{\alpha}\left(\boldsymbol{\varphi}_{h}\right) \cdot \delta \boldsymbol{\varphi}_{, \alpha} d \mathcal{A}_{0}+\int_{\mathcal{A}_{h}} \bar{j} \boldsymbol{l} \cdot \delta \boldsymbol{t} \lambda_{h} d \mathcal{A}_{0}+ \\
& \int_{\mathcal{A}_{h}} \bar{j} \tilde{\boldsymbol{m}}^{\alpha}\left(\boldsymbol{\varphi}_{h}\right) \cdot\left(\delta \boldsymbol{t} \lambda_{h}\right)_{, \alpha} d \mathcal{A}_{0}+
\end{aligned}
$$

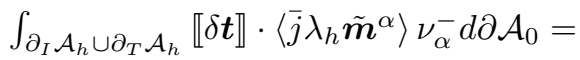

$$
\begin{aligned}
& \int_{\partial_{N} \mathcal{A}_{h}} \bar{j} \overline{\boldsymbol{n}} \cdot \delta \boldsymbol{\varphi} d \mathcal{A}_{0}+\int_{\partial_{M} \mathcal{A}_{h}} \bar{j} \overline{\tilde{\boldsymbol{m}}} \cdot \delta \boldsymbol{t} \lambda_{h} d \mathcal{A}_{0}+ \\
& \int_{\mathcal{A}_{h}} \boldsymbol{n}^{\mathcal{A}_{0}} \cdot \delta \boldsymbol{\varphi} \bar{j} d \mathcal{A}_{0}+\int_{\mathcal{A}_{h}} \tilde{\boldsymbol{m}}^{\mathcal{A}_{0}} \cdot \delta \boldsymbol{t} \lambda_{h} \bar{j} d \mathcal{A}_{0} .
\end{aligned}
$$

Although this formation is consistent, it is nor stable neither verifying the compatibility condition $\llbracket t \rrbracket=0$ on the interior domain definition. Toward this end, symmetrization and quadratic terms are added, see [10] for details. This quadratic term depends on a stabilization parameter $\beta$ that has to be chosen large enough to lead to a stable weak statement of the problem. Therefore, after adding such contributions, the final weak statement of the problem is

$$
\begin{aligned}
& \int_{\mathcal{A}_{h}} \bar{j} \boldsymbol{n}^{\alpha}\left(\boldsymbol{\varphi}_{h}\right) \cdot \delta \boldsymbol{\varphi}_{, \alpha} d \mathcal{A}_{0}+\int_{\mathcal{A}_{h}} \bar{j} \boldsymbol{l} \cdot \delta \boldsymbol{t} \lambda_{h} d \mathcal{A}_{0}+ \\
& \int_{\mathcal{A}_{h}} \bar{j} \tilde{\boldsymbol{m}}^{\alpha}\left(\boldsymbol{\varphi}_{h}\right) \cdot\left(\delta \boldsymbol{t} \lambda_{h}\right)_{, \alpha} d \mathcal{A}_{0}+
\end{aligned}
$$

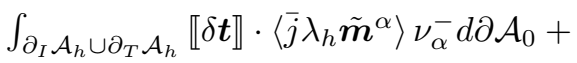

$$
\begin{aligned}
& \int_{\partial_{I} \mathcal{A}_{h} \cup \partial_{T} \mathcal{A}_{h}} \llbracket \boldsymbol{t}\left(\boldsymbol{\varphi}_{h}\right) \rrbracket \cdot\left\langle\overline { j } _ { 0 } \mathcal { H } _ { m } ^ { \alpha \beta \gamma \delta } \left(\delta \boldsymbol{\varphi}_{, \gamma} \cdot \boldsymbol{t}_{, \delta}+\right.\right. \\
& \left.\left.\boldsymbol{\varphi}_{, \gamma} \cdot \delta \boldsymbol{t}_{, \delta}\right) \boldsymbol{\varphi}, \beta+\bar{j} \lambda_{h} \tilde{\boldsymbol{m}}^{\alpha} \cdot \boldsymbol{\varphi}_{, \beta} \quad \delta \boldsymbol{\varphi}, \beta\right\rangle \nu_{\alpha}^{-} d \partial \mathcal{A}_{0}+ \\
& \int_{\partial_{I} \mathcal{A}_{h} \cup \partial_{T} \mathcal{A}_{h}} \llbracket \boldsymbol{t}\left(\boldsymbol{\varphi}_{h}\right) \rrbracket \cdot \boldsymbol{\varphi}_{, \beta}\left\langle\frac{\beta \bar{j}_{0} \mathcal{H}_{m}^{\alpha \beta \gamma \delta}}{h^{s}}\right\rangle \\
& \llbracket \delta \boldsymbol{t} \rrbracket \cdot \boldsymbol{\varphi}_{, \gamma} \nu_{\alpha}^{-} \nu_{\delta}^{-} d \partial \mathcal{A}_{0} \\
& =\int_{\partial_{N} \mathcal{A}_{h}} \bar{j} \overline{\boldsymbol{n}} \cdot \delta \boldsymbol{\varphi} d \mathcal{A}_{0}+\int_{\partial_{M} \mathcal{A}_{h}} \bar{j} \overline{\tilde{\boldsymbol{m}}} \cdot \delta \boldsymbol{t} \lambda_{h} d \mathcal{A}_{0}+ \\
& \int_{\mathcal{A}_{h}} \boldsymbol{n}^{\mathcal{A}_{0}} \cdot \delta \boldsymbol{\varphi} \bar{j} d \mathcal{A}_{0}+\int_{\mathcal{A}_{h}} \tilde{\boldsymbol{m}}^{\mathcal{A}_{0}} \cdot \delta \boldsymbol{t} \lambda_{h} \bar{j} d \mathcal{A}_{0},
\end{aligned}
$$

which enforces weakly the condition $\llbracket t \rrbracket=0$. In this last equation, the linearized bending tangent moduli

$$
\begin{aligned}
\mathcal{H}_{m}^{\alpha \beta \gamma \delta}= & \frac{E\left(h_{\max }-h_{\min }\right)^{3}}{12\left(1-\nu^{2}\right)}\left[\nu \boldsymbol{\varphi}_{0}^{, \alpha} \cdot \boldsymbol{\varphi}_{0}^{, \beta} \quad \boldsymbol{\varphi}_{0}^{, \gamma} \cdot \boldsymbol{\varphi}_{0}^{, \delta}+\right. \\
& \frac{1}{2}(1-\nu) \boldsymbol{\varphi}_{0}^{, \alpha} \cdot \boldsymbol{\varphi}_{0}^{, \gamma} \boldsymbol{\varphi}_{0}^{, \delta} \cdot \boldsymbol{\varphi}_{0}^{, \beta}+ \\
& \frac{1}{2}(1-\nu) \boldsymbol{\varphi}_{0}^{, \alpha} \cdot \boldsymbol{\varphi}_{0}^{, \delta} \quad \boldsymbol{\varphi}_{0}^{, \gamma} \cdot \boldsymbol{\varphi}_{0}^{, \beta}
\end{aligned}
$$

are used in order to ensure the stability of the formulation for any possible constitutive model, see [4] for discussion.

\section{NUMERICAL APPLICATION}

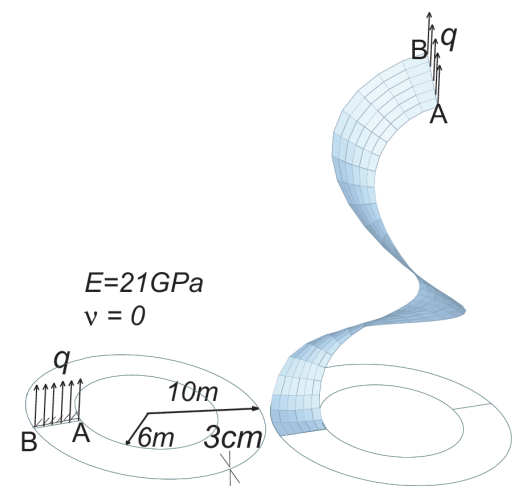

Figure 2: Study of the thin plate ring: a) Geometry of the initially cut ring (one side of line $A B$ is clamped and the other one is uniformly loaded. b) Final deformation of the test for a regular mesh of 16 bi-quadratic quadrangular elements on the circumference and 3 bi-quadratic quadrangular elements on the edge $A B$.

This example consists into a thin plate ring cut along a radius AB. On one side of this cutting, the plate is clamped, while a uniform vertical loading $q$ is applied on the other side, see Figure 2a. This test has widely been used in the 
literature to compare shells formulations when large rotations arise.

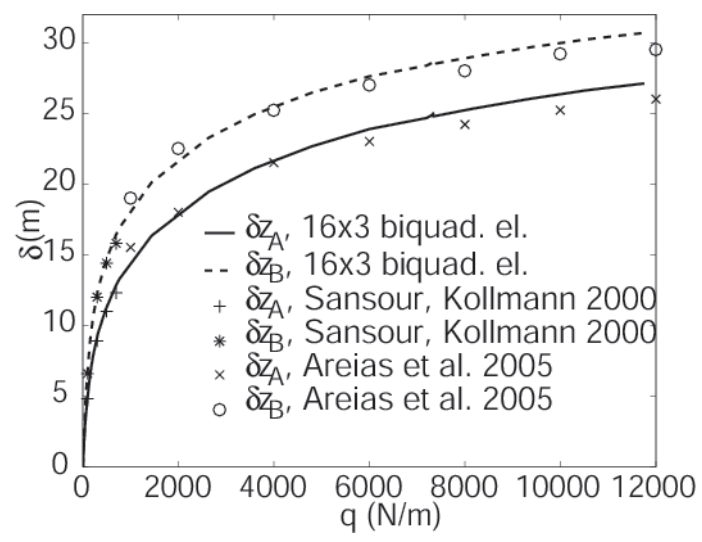

Figure 3: Study of the plate ring: force-displacement evolutions of the nodes located at the cutting. Stabilization parameter $\beta=10^{2}$.

This simulation is computed using the proposed discontinuous Galerkin formulation applied to 4-Gauss-point 9node bi-quadratic elements. The final deformed configuration is illustrated in Figure $2 b$, and the displacement evolutions of nodes A and B located at the cutting are shown in Figure 3. Although the mesh experiences large distortion during the deformation process, the solution is in good agreement with the ones obtained in the literature, and in particular with: (i) The hybrid stress formulation proposed by Sansour and Kollmann [12], for whom results are displayed for $q<3000 \mathrm{~N} \cdot \mathrm{m}^{-1}$ (which is the maximum loading considered in this reference). (ii) The mixed formulation based on mid-side rotations proposed by Areias et al. [13], which converges for an applied linear force reaching $12000 \mathrm{~N} \cdot \mathrm{m}^{-1}$.

\section{CONCLUSIONS}

A general DG formulation of linear Kirchhoff-Love shells for finite deformations has been presented. When establishing this weak form, the discontinuities in the displacement derivative between two elements are accounted for by recurse to a DG method, leading to a one-field formulation.

\section{REFERENCES}

[1] MGD Geers, EWC Coenen, and VG Kouznetsova. Multi-scale computational homogenization of structured thin sheets. Modelling and Simulation in Materials Science and Engineering, 15:S393-S404, 2007.

[2] A. Ten Eyck and A. Lew. Discontinuous Galerkin methods for non-linear elasticity. International Journal for Numerical Methods in Engineering, 67: 1204-1243, 2006.

[3] L. Noels and R. Radovitzky. A general discontinuous Galerkin method for finite hyperelasticity. Formulation and numerical applications. International Journal for Numerical Methods in Engineering, 68(1):64-97, 2006.

[4] L. Noels and R. Radovitzky. An explicit discontinuous Galerkin method for non-linear solid dynamics. Formulation, parallel implementation and scalability properties. International Journal for Numerical Methods in Engineering, 74:1393-1420, 2008.

[5] A. Ten Eyck, A. Celiker, and A. Lew. Adaptive stabilization of discontinuous galerkin methods for nonlinear elasticity: Motivation, formulation and numerical examples. Computer Methods in Applied Mechanics and Engineering, 197:-, 2008.

[6] G Engel, K. Garikipati, T.J.R. Hughes, M.G. Larson, L Mazzei, and RL Taylor. Continuous/discontinuous finite element approximations of fourth-order elliptic problems in structural and continuum mechanics with applications to thin beams and plates. Computer Methods in Applied Mechanics and Engineering, 191:3669-3750, 2002.

[7] P. Hansbo and M.G. Larson. A discontinuous Galerkin method for the plate equation. CALCOLO, 39:41-59, 2002.

[8] G.N. Wells and N.T. Dung. A $C^{0}$ discontinuous Galerkin formulation for Kirchhoff plates. Computer Methods in Applied Mechanics and Engineering, 196:3370-3380, 2007.

[9] L. Noels and R. Radovitzky. A new discontinuous Galerkin method for kirchhoff-love shells. Computer Methods in Applied Mechanics and Engineering, 197:2901-2929, 2008.

[10] L. Noels. A discontinuous galerkin formulation of non-linear kirchhoff-love shells. International Journal for Numerical Methods in Engineering, page Accepted, 2008.

[11] J.C. Simo and D.D. Fox. On a stress resultant geometrically exact shell model. Part I: formulation and optimal parametrization. Computer Methods in Applied Mechanics and Engineering, 72:267-304, 1989.

[12] C. Sansour and F.G. Kollman. Families of 4-node and 9-node finite elements for a finite deformation shell theory. An assessment of hybrid stress, hybrid strain and enhanced strain elements. Computational Mechanics, 24:435-447, 2000.

[13] P.M.A. Areias, J.-H. Song, and T. Belytschko. A finite strain quadrilateral shell element based on discrete kirchhoff-love constraints. International Journal for Numerical Methods in Engineering, 64: 1166-1206, 2005. 